\title{
Awareness of medical students about standard isolation precautions In Sulaimani City
}

\author{
Bushra M. Ali \\ Family \& Community Medicine Dep. \\ College of Medicine \\ Sulaimani University \\ Sulaimani / Iraq \\ bmakfb.2002@yahoo.come \\ Balen Tayb Abdullah \\ College of medicine \\ Sulaimani University \\ Sulaimani, Iraq \\ balentayb@gmail.com
}

\author{
Saman A. Mohammed \\ Family \& Community Medicine Dep. \\ College of Medicine \\ Sulaimani University \\ Sulaimani / Iraq \\ dr.saman_am@yahoo.com \\ Osama Muhammad \\ College of medicine \\ Sulaimani University \\ Sulaimani, Iraq \\ Osama.mohammed99999@gmail.com
}

\author{
Muhammad Rauf \\ College of medicine \\ Sulaimani University \\ Sulaimani, Iraq \\ Star.sky1353@gmail.com
}

Volume 4 - Special Issue: 3rd International Conference on Health \& Medical Sciences: Insight into Advanced Medical Research (ICHMS 2019)

DOI:

10.24017/science.2019

.ICHMS.21

Received:

14 June 2019

Accepted:

7 July 2019

\section{Abstract}

The objectives of this study are to assess knowledge, Attitude and practice of medical students about standard isolation precautions and different college students and to identify those who vaccinated with $\mathrm{HBV}$ vaccine. A cross-sectional study was conducted from 27th of February 2019 till 1st of March 2019 in Sulaimani University among medical students .A convenient sample of 401 students were participated. Data was collected via online, entered by excel program and analyzed by SPSS program version 21. Chi-square test used to get the association, $P$ value which is equal or less than 0.05 was regarded statistically significant. Majority of medical students had accepted knowledge (67\%), their attitude was positive (70 \%), and had low practice (35\%). Most of the students answered wrongly regarding gloves should be worn if they attached to mucous membrane or non-intact skin and the highest percentage was among nursing college (85.3\%), there was a significant association regarding $P$ value (0.035).Most of the students believed that all health providers are at risk of occupational infections (75.6\%), and the highest percentage was among nursing college 
(94.1\%) and there was a significant association regarding $P$ value (0.006). The percentage of the students that have taken a vaccine for Hepatitis $B$ is (56.4\%). Most of students had good knowledge regarding all aspects of Isolation Precaution, they had appropriate attitude toward it, high rate of medical students were lacking practice regarding the subject, in average college of Nursing had highest rate of all correct answers, About half of medical students have taken a vaccine for Hepatitis $B$.

Keywords: Standard isolation precautions, Medical students, Sulaimani

\section{INTRODUCTION}

Minimizing pathogens transmission risk from recognized and unrecognized sources is known as standard precautions, they are the basic level of infection control precautions which are to be used, as a minimum, in the care of all patients ${ }^{1}$. Medical staff and medical students are at risk of getting infection through occupational exposure. The infection can also transmitted from health workers to the patients and other office workers ${ }^{2}$. The risk of transmission of HIV to the health care worker when accidentally exposed to the needle stick or other sharps injury is $\% 5^{3}$. General precautions comprise of (hand hygiene, the use of PPE, respiratory hygiene/cough etiquette, environmental cleaning, prevention of needle stick injuries and safe waste management) ${ }^{4}$. Through adherence to standard precautions, risk reduction must be undertaken for all blood borne pathogens by appropriate use of safety devices, using personal protective equipment, and providing a needle disposal system in work place ${ }^{5}$. A percentage of these risks can be prevented through a strict adherence by health staff to standard precaution ${ }^{6}$. Medical students before their initial training period at hospital should have adequate knowledge which is a pre-requisite for compliance. In addition to that a health care student must be trained a specialized training before undertakes any patient procedure involving sharp devices $^{7}$ a few studies have reported on medical student knowledge of standard precaution and isolation noted that lack of adequate of information in standard isolation and precaution ${ }^{8}$. The observance of hygiene recommendations by students is reported as being weak, medical students rarely wash their hands after examining patients ${ }^{9}$. All the staff with potential exposure to blood and blood products must be educated regarding transmission mechanisms and preventive measures of the infectious diseases ${ }^{10}$. Knowledge, attitude, and practice of medical students towards standard isolation precautions were not appropriate these were found in several studies on the efficacy of this concept have been published. In Iran there are limited studies in relation to Iranian medical students towards standard isolation precautions.

\section{Objectives:}

1- To identify the awareness of the medical students about standard isolation precautions.

2- To find out the associations between different medical colleges students regarding knowledge, Attitude and practice about standard isolation precautions.

3- To identify difference between collages regarding receiving hepatitis B vaccine. 


\section{METHODOLOGY}

Study design: Cross-sectional study.

Study duration: For five days, study began in 27th February 2019 and ended in 1st March 2019.

Study setting: The study was conducted in Sulaimani University, Faculty of Medical Science (Colleges of Medicine, Dentistry, Pharmacy and Nursing).

Sampling method and Sample size: A convenient sampling type was conducted. According to responses from online Google forms, 401 medical students from all colleges that were included have filled the form and retuned back online. A self- administrative questionnaire was used to collect the information which was composed of four sections: Socio-demographic information: It included (Age, Gender, Marital states, Residency, Economic state, College) of the participants. Evaluation of students' knowledge: It includes 10 questions to determine their knowledge about isolation precautions. Evaluation of students' attitude: It includes 10 questions to estimate thoughts and attitude of students regarding the topic. Evaluation of students' practice: It includes 9 questions to evaluate practical activities of students in the practice sections. Data was collected via online questionnaire then entered to Google form screen sheet, transmitted into Microsoft Excel version 2016, after that into SPSS Version 21. And two approaches had been made: I. Descriptive approach, to calculate Frequency, Percentage, Mean, Standard Deviation, and Diagrams. II. Analytical approach, to find association between variables using Chi-square (with P-value less than or equal to0.05 as statistically significant).

Ethical consideration: A permission was taken from department of Family and community medicine/college of medicine /Sulaimani University. It was protected legally by informed consent, and participants were informed that they are free to fill the form.

\section{RESULTS}

Mean age/ year of participants was (21.33 $\pm 1.89 S D)$

Table (1) shows the highest frequency of participation was among females which was (50.4\%) which is too close to male participation (49.6\%), and among college of medicine which was (60.8\%), Regarding to residency participation among once live inside city was higher which was (53.9\%), (89.3\%) of participants were single ,while $(78.3 \%)$ had a moderate economic state.

Table 1: distribution of medical students according to their socio-demographic

$$
\text { states }
$$

\begin{tabular}{|l|c|c|c|}
\hline \multicolumn{2}{|c|}{ Socio-demographic } & Frequency & Percentage (\%) \\
\hline \multirow{3}{*}{ Gender } & Female & 202 & 50.4 \\
\cline { 2 - 4 } & Male & 199 & 49.6 \\
\hline \multirow{2}{*}{ Marital states } & Divorced & 9 & 2.2 \\
\cline { 2 - 4 } & Married & 34 & 8.5 \\
\hline
\end{tabular}




\begin{tabular}{|c|c|c|c|}
\cline { 2 - 4 } & Single & 358 & 89.3 \\
\hline \multirow{3}{*}{ Residency } & Inside city & 216 & 53.9 \\
\cline { 2 - 4 } & Outside city & 185 & 46.1 \\
\hline \multirow{3}{*}{ Economic state } & High & 42 & 10.5 \\
\cline { 2 - 4 } & Low & 45 & 11.2 \\
\cline { 2 - 4 } & Moderate & 314 & 78.3 \\
\hline \multirow{2}{*}{ College } & Dentistry & 79 & 19.7 \\
\cline { 2 - 4 } & Medicine & 244 & 60.8 \\
\cline { 2 - 4 } & Nursery & 34 & 8.5 \\
\cline { 2 - 4 } & Pharmacy & 44 & 11.0 \\
\hline Total & & 401 & 100.0 \\
\hline
\end{tabular}

Table (2) shows the associations between deferent colleges regarding their Knowledge about prevention of standard precautions, by asking about after any contact with secretions, contaminated item, blood and bloody fluid hands should be washed, $80.5 \%$ said yes with a high proportion among medicine which $82.4 \%$ of them answered yes with statistically not significant p value (0.687), And regarding touching mucous membranes or non-intact skin, wearing of gloves should be done $79.3 \%$ said yes with a high proportion in college of nursing that $85.3 \%$ of them said yes and with a significant p value (0.035), About $68.1 \%$ said they should wear protective eyeglasses to protect mucous membranes of the eyes and among them 85.3\% from nursing with non-significant $\mathrm{p}$ value $(0.263)$, Regarding the surgical mask $79.6 \%$ said yes to protect the mouth and nose from invasive procedures and activities surgical mask should be worn, which among them highest percentage was in collage of nursing $85.3 \%$ with statistically not significant p value (0.725), by asking Cleaning And disinfect-ting should be applied for any common equipment between patients who are on contact precautions 76.3 of participants said yes which among them highest percentage was in college of pharmacy $86.4 \%$ with statistically not significant p value (0.389), regarding Wearing gown is necessary when entering patient's room on contact precautions if you anticipate contact with patient or environment.

Table 2: associations between deferent colleges regarding their Knowledge about prevention of standard precautions

\begin{tabular}{|c|c|c|c|c|c|c|c|}
\hline \multirow{3}{*}{\multicolumn{2}{|c|}{$\begin{array}{l}\text { Knowledge about } \\
\text { Prevention }\end{array}$}} & \multicolumn{4}{|c|}{ College } & \multirow{2}{*}{ Total } & \multirow{3}{*}{$\begin{array}{c}\text { P. } \\
\text { Value }\end{array}$} \\
\hline & & \multirow{2}{*}{$\begin{array}{c}\text { Medicine } \\
\text { Number } \\
(\%)\end{array}$} & \multirow{2}{*}{$\begin{array}{c}\text { Nursery } \\
\text { Number } \\
\text { (\%) }\end{array}$} & \multirow{2}{*}{$\begin{array}{c}\text { Dentistry } \\
\text { Number } \\
\text { (\%) }\end{array}$} & \multirow{2}{*}{$\begin{array}{c}\text { Pharmacy } \\
\text { Number } \\
(\%)\end{array}$} & & \\
\hline & & & & & & $\begin{array}{c}\text { Number } \\
(\%)\end{array}$ & \\
\hline \multirow{3}{*}{$\begin{array}{l}\text { 1. Should hands be } \\
\text { washed after } \\
\text { accidental contact } \\
\text { with blood bloody } \\
\text { fluid, secretions }\end{array}$} & I don't know & $8(3.3)$ & $1(2.9)$ & $6(7.6)$ & $3(6.8)$ & $18(4.5)$ & \multirow{3}{*}{0.687} \\
\hline & No & $35(14.3)$ & $6(17.6)$ & 13(16.5) & $6(13.6)$ & $60(15.0)$ & \\
\hline & Yes & 201(82.4) & 27(79.4) & $60(75.9)$ & 35(79.5) & $323(80.5)$ & \\
\hline
\end{tabular}




\begin{tabular}{|c|c|c|c|c|c|c|c|}
\hline $\begin{array}{l}\text { contaminated } \\
\text { item? }\end{array}$ & Total & $244(100)$ & $34(100)$ & $79(100)$ & $44(100)$ & 401(100) & \\
\hline \multirow{4}{*}{$\begin{array}{l}\text { 2. should gloves be } \\
\text { worn when touching } \\
\text { mucous membranes } \\
\text { or non-intact skin? }\end{array}$} & I don't know & $19(7.8)$ & $2(5.9)$ & $5(6.3)$ & $6(13.6)$ & $32(8.0)$ & \multirow{4}{*}{0.035} \\
\hline & No & $25(10.2)$ & $3(8.8)$ & $19(24.1)$ & $4(9.1)$ & $51(12.7)$ & \\
\hline & Yes & $200(82.0)$ & 29(85.3) & $55(69.6)$ & $34(77.3)$ & $318(79.3)$ & \\
\hline & Total & $244(100)$ & $34(100)$ & $79(100)$ & $44(100)$ & 401(100) & \\
\hline \multirow{4}{*}{$\begin{array}{l}\text { 3. Goggles } \\
\text { (Protective } \\
\text { eyeglasses) should } \\
\text { be worn to protect } \\
\text { mucous membranes } \\
\text { of the eyes }\end{array}$} & I don't know & $34(13.9)$ & $3(8.8)$ & $13(16.5)$ & $10(22.7)$ & $60(15.0)$ & \multirow{4}{*}{0.263} \\
\hline & No & $44(18.0)$ & $2(5.9)$ & 14(17.7) & $8(18.2)$ & $68(17.0)$ & \\
\hline & Yes & $166(68.0)$ & $29(85.3)$ & $52(65.8)$ & $26(59.1)$ & $273(68.1)$ & \\
\hline & Total & $244(100)$ & $34(100)$ & $79(100)$ & $44(100)$ & 401(100) & \\
\hline \multirow{4}{*}{$\begin{array}{l}\text { 4. A surgical mask } \\
\text { should be worn to } \\
\text { protect the nose and } \\
\text { mouth from } \\
\text { invasive } \\
\text { procedures and } \\
\text { activities }\end{array}$} & I don't know & $19(7.8)$ & $3(8.8)$ & $5(6.3)$ & $4(9.1)$ & $31(7.7)$ & \multirow{4}{*}{0.725} \\
\hline & No & 29(11.9) & $2(5.9)$ & $14(17.7)$ & $6(13.6)$ & $51(12.7)$ & \\
\hline & Yes & 196(80.3) & 29(85.3) & $60(75.9)$ & $34(77.3)$ & 319(79.6) & \\
\hline & Total & $244(100)$ & $34(100)$ & $79(100)$ & $44(100)$ & 401(100) & \\
\hline \multirow{4}{*}{$\begin{array}{l}\text { 5. Cleaning And } \\
\text { disinfect-ting should } \\
\text { be applied for any } \\
\text { common equipment } \\
\text { between patients who } \\
\text { are on } \\
\text { contact } \\
\text { precautions }\end{array}$} & I don't know & $25(10.2)$ & $6(17.6)$ & $9(11.4)$ & $3(6.8)$ & $43(10.7)$ & \multirow{4}{*}{0.389} \\
\hline & No & $30(12.3)$ & $6(17.6)$ & $13(16.5)$ & $3(6.8)$ & $52(13.0)$ & \\
\hline & Yes & 189(77.5) & $22(64.7)$ & $57(72.2)$ & 38(86.4) & $306(76.3)$ & \\
\hline & Total & $244(100)$ & $34(100)$ & $79(100)$ & $44(100)$ & 401(100) & \\
\hline \multirow{4}{*}{$\begin{array}{l}\text { 6. Wearing gown } \\
\text { is necessary when } \\
\text { entering patient's room } \\
\text { on Contact precautions } \\
\text { if } \\
\text { you anticipate contact } \\
\text { with patient or } \\
\text { Environment. }\end{array}$} & $\begin{array}{l}\text { I don't } \\
\text { know }\end{array}$ & $44(18.0)$ & $4(11.8)$ & $10(12.7)$ & $9(20.5)$ & $67(16.7)$ & \multirow[t]{4}{*}{0.844} \\
\hline & \begin{tabular}{l|l} 
No \\
s
\end{tabular} & $39(16.0)$ & $7(20.6)$ & 13(16.5) & 6(13.6) & $65(16.2)$ & \\
\hline & Yes & 161(66.0) & 23(67.6) & $56(70.9)$ & 29(65.9) & $269(67.1)$ & \\
\hline & Total & $244(100)$ & $34(100)$ & $79(100)$ & $44(100)$ & $401(100)$ & \\
\hline
\end{tabular}

Table (3) shows the associations between deferent colleges regarding their Knowledge about general information of standard precautions, about health providers include radiologists $57.1 \%$ said yes they are with highest risk of exposure to tuberculosis and the highest percentage was in college of pharmacy $65.9 \%$ with not-significant p. value (0.387),regarding needles should be bent before disposal $48.1 \%$ of them said yes with highest percentage in college of medicine which $51.6 \%$ with not-significant p value (0.152). Only $29.7 \%$ of participants said no regarding routine immunization for healthcare providers include Rabies, HIV and Rubella in which the highest percentage was in collage of dentistry $31.6 \%$ with not-significant p value (0.951). 
Table 3: associations between deferent colleges regarding their Knowledge about general information of standard precautions

\begin{tabular}{|c|c|c|c|c|c|c|c|}
\hline \multirow{3}{*}{\multicolumn{2}{|c|}{$\begin{array}{l}\text { Knowledge about } \\
\text { general information }\end{array}$}} & \multicolumn{4}{|c|}{ College } & \multirow{3}{*}{$\begin{array}{c}\text { Total } \\
\begin{array}{c}\text { Number } \\
(\%)\end{array}\end{array}$} & \multirow{3}{*}{$\begin{array}{c}\text { P. } \\
\text { Value }\end{array}$} \\
\hline & & \multirow{2}{*}{$\begin{array}{c}\text { Medicine } \\
\text { Number } \\
\text { (\%) }\end{array}$} & \multirow{2}{*}{$\begin{array}{c}\text { Nursery } \\
\text { Number } \\
\text { (\%) }\end{array}$} & \multirow{2}{*}{$\begin{array}{c}\text { Dentistry } \\
\text { Number } \\
\text { (\%) }\end{array}$} & \multirow{2}{*}{$\begin{array}{c}\text { Pharmacy } \\
\text { Number (\%) }\end{array}$} & & \\
\hline & & & & & & & \\
\hline \multirow{4}{*}{$\begin{array}{l}\text { 1. Health providers } \\
\text { with highest risk of } \\
\text { exposure to } \\
\text { tuberculosis } \\
\text { include } \\
\text { radiologists. }\end{array}$} & $\begin{array}{l}\text { I don't } \\
\text { know }\end{array}$ & 69(28.3) & $6(17.6)$ & $14(17.7)$ & $9(20.5)$ & $98(24.4)$ & \multirow{4}{*}{0.387} \\
\hline & No & $44(18.0)$ & $7(20.6)$ & $17(21.5)$ & $6(13.6)$ & 74(18.5) & \\
\hline & Yes & 131(53.7) & $21(61.8)$ & $48(60.8)$ & $29(65.9)$ & $229(57.1)$ & \\
\hline & Total & $244(100)$ & $34(100)$ & $79(100)$ & $44(100)$ & 401(100) & \\
\hline \multirow[t]{4}{*}{$\begin{array}{l}\text { 2. needle should be } \\
\text { bent before } \\
\text { disposal }\end{array}$} & $\begin{array}{l}\text { I don't } \\
\text { know }\end{array}$ & 58(23.8) & $14(41.2)$ & $18(22.8)$ & $9(20.5)$ & $99(24.7)$ & \multirow{4}{*}{0.152} \\
\hline & No & $60(24.6)$ & $7(20.6)$ & $27(34.2)$ & 15(34.1) & 109(27.2) & \\
\hline & Yes & 126(51.6) & 13(38.2) & $34(43.0)$ & $20(45.5)$ & 193(48.1) & \\
\hline & Total & $244(100)$ & $34(100)$ & $79(100)$ & $44(100)$ & 401(100) & \\
\hline \multirow{4}{*}{$\begin{array}{l}\text { 3. Routine } \\
\text { immunizations for } \\
\text { healthcare } \\
\text { providers include } \\
\text { HIV, rubella and } \\
\text { rabies }\end{array}$} & $\begin{array}{l}\text { I don't } \\
\text { know }\end{array}$ & $49(20.1)$ & $9(26.5)$ & 14(17.7) & $9(20.3)$ & $81(20.2)$ & \multirow{4}{*}{0.951} \\
\hline & No & 74(30.3) & 8(23.5) & 25(31.6) & $12(27.3)$ & 119(29.7) & \\
\hline & Yes & $121(49.6)$ & $17(50.0)$ & $40(50.6)$ & $23(52.3)$ & 201(50.1) & \\
\hline & Total & 244(100) & 34(100) & 79(100) & $44(100)$ & 401(100) & \\
\hline
\end{tabular}

Fig (1) About 56.9\% of participants said all patients can be regarded as sources of infection whatever are their diagnosis and 33.2\% said no only $10.0 \%$ said I don't know and the highest proportion was in college of nursing which $76.5 \%$ of students said yes and lowest in college of medicine which $51.2 \%$ of them said yes with not-significant $p$ value $(0.113)$. 


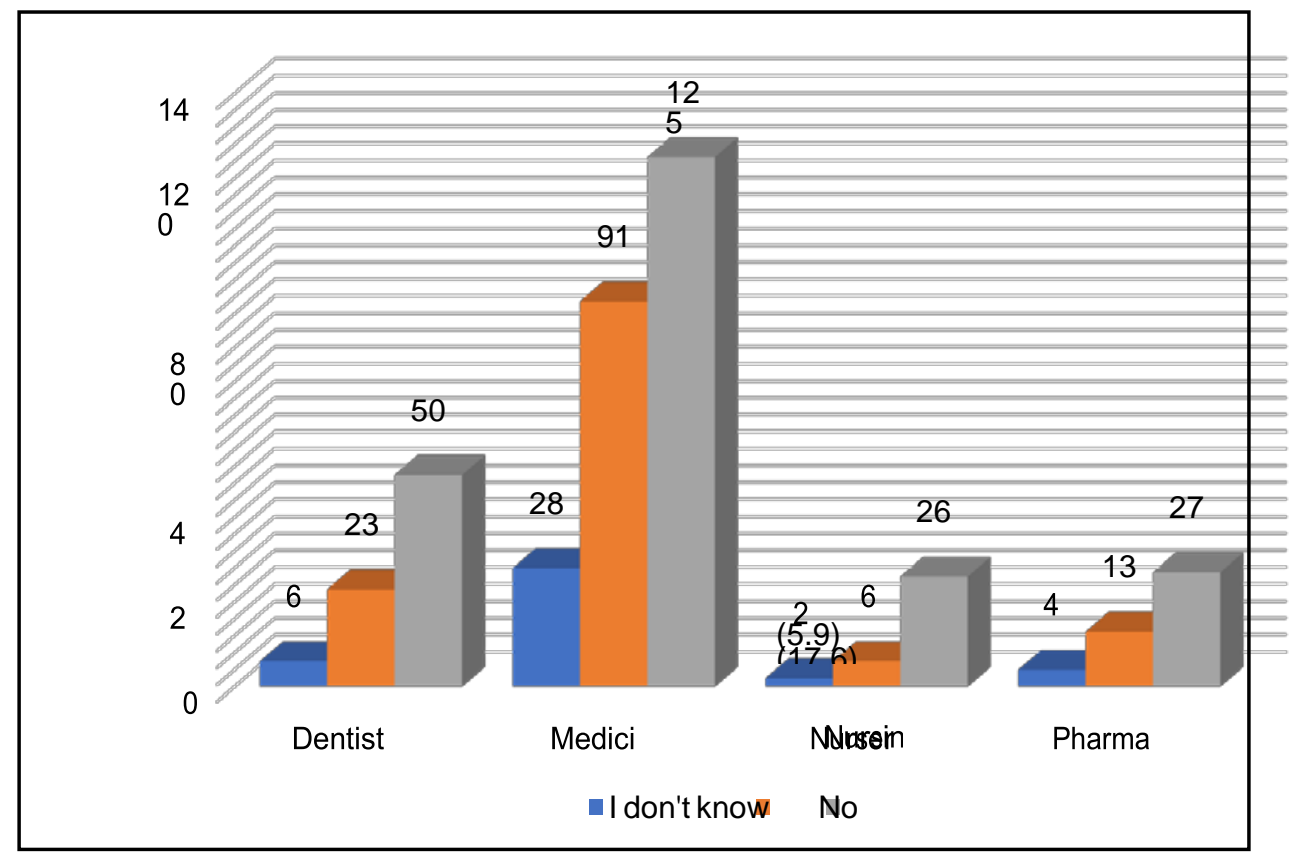

Figure 1: Showing the difference between students in different collages regarding answering that all patients are sources of infection regardless their diagnosis.

Table (4) shows the associations between deferent colleges regarding their attitude of standard precautions, $75.6 \%$ believe that all healthcare workers are at risk of infections during their work and among them highest percentage was in collage of Nursing $94.1 \%$ with statistically high significant $\mathrm{p}$ value (0.006), by asking do they think hand washing can be replaced by using of gloves $52.4 \%$ said yes while among them highest percentage was in college of pharmacy $72.7 \%$ of them said yes with significant p value (0.023), by asking do they believe Health- care providers should be tested annually by tuberculin skin test $72.3 \%$ said yes and among them highest percentage was in college of pharmacy $79.5 \%$ of them said yes with nonsignificant $\mathrm{p}$ value $(0.223)$, when we ask did they think Health-care providers should receive annual influenza vaccine $75.3 \%$ said yes which among them highest percentage was in collage of medicine $77.5 \%$ of them said yes with non-significant p value (0.539), and by asking do they think masks which composed of cotton are most protective $67.3 \%$ said yes and among them highest percentage was in collage of nursing $79.4 \%$ and statistically non-significant $\mathrm{p}$ value (0.261), by asking do they think on the same patient between different procedures gloves should be changed $75.1 \%$ said yes which among them the highest percentage was in collage of medicine $79.5 \%$ with a statistically non-significant $\mathrm{p}$ value (0.19),by asking, do they think Gloves and masks after proper cleaning can be re-used 51.4\% said no and among them the highest percentage was in college of dentistry $57.0 \%$ and statistically non-significant p value (0.53), when we asked do they think only sweat not regarded as source of infection $75.6 \%$ of participants said yes and among them highest percentage was in collage of nursing $82.4 \%$ with non-significant $\mathrm{p}$ value (0.742),and by asking do they think Patients with a disease that needs contact precautions must be kept in a private room $77.8 \%$ of participants said yes which among them highest percentage was in collage of nursing $91.2 \%$ and statistically nonsignificant p value (0.165). 
Table 4: associations between deferent colleges regarding their attitude of standard precautions

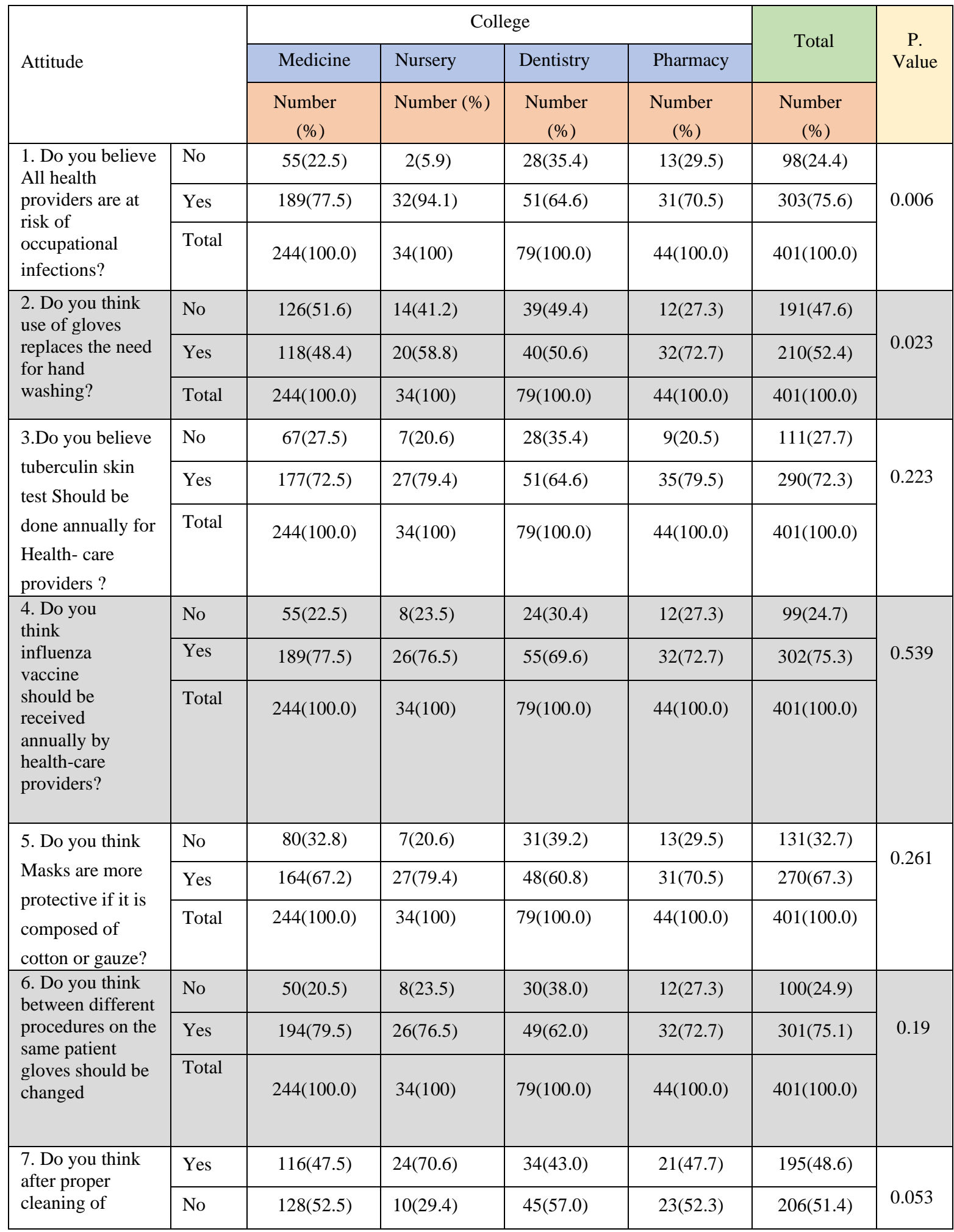




\begin{tabular}{|c|c|c|c|c|c|c|c|}
\hline $\begin{array}{l}\text { masks and } \\
\text { gloves we can re- } \\
\text { use them }\end{array}$ & Total & $244(100.0)$ & $34(100)$ & $79(100.0)$ & $44(100.0)$ & $401(100.0)$ & \\
\hline \multirow{3}{*}{$\begin{array}{l}\text { 8. Do you think } \\
\text { that only sweat } \\
\text { shouldn't be } \\
\text { regarded as } \\
\text { sources } \\
\text { of infection? }\end{array}$} & No & $59(24.2)$ & $6(17.6)$ & 21(26.6) & 12(27.3) & $98(24.4)$ & \multirow{3}{*}{0.742} \\
\hline & Yes & 185(75.8) & 28(82.4) & $58(73.4)$ & $32(72.7)$ & $303(75.6)$ & \\
\hline & Total & $244(100.0)$ & $34(100)$ & $79(100.0)$ & $44(100.0)$ & $401(100.0)$ & \\
\hline
\end{tabular}

Fig. (2) by asking did they think Hand washing reduces the incidence of healthcarerelated infections $81.2 \%$ of them said yes, which among them highest percentage was in collage of medicine which’s $86.5 \%$ and statistically highly significant p value $(0.008)$.

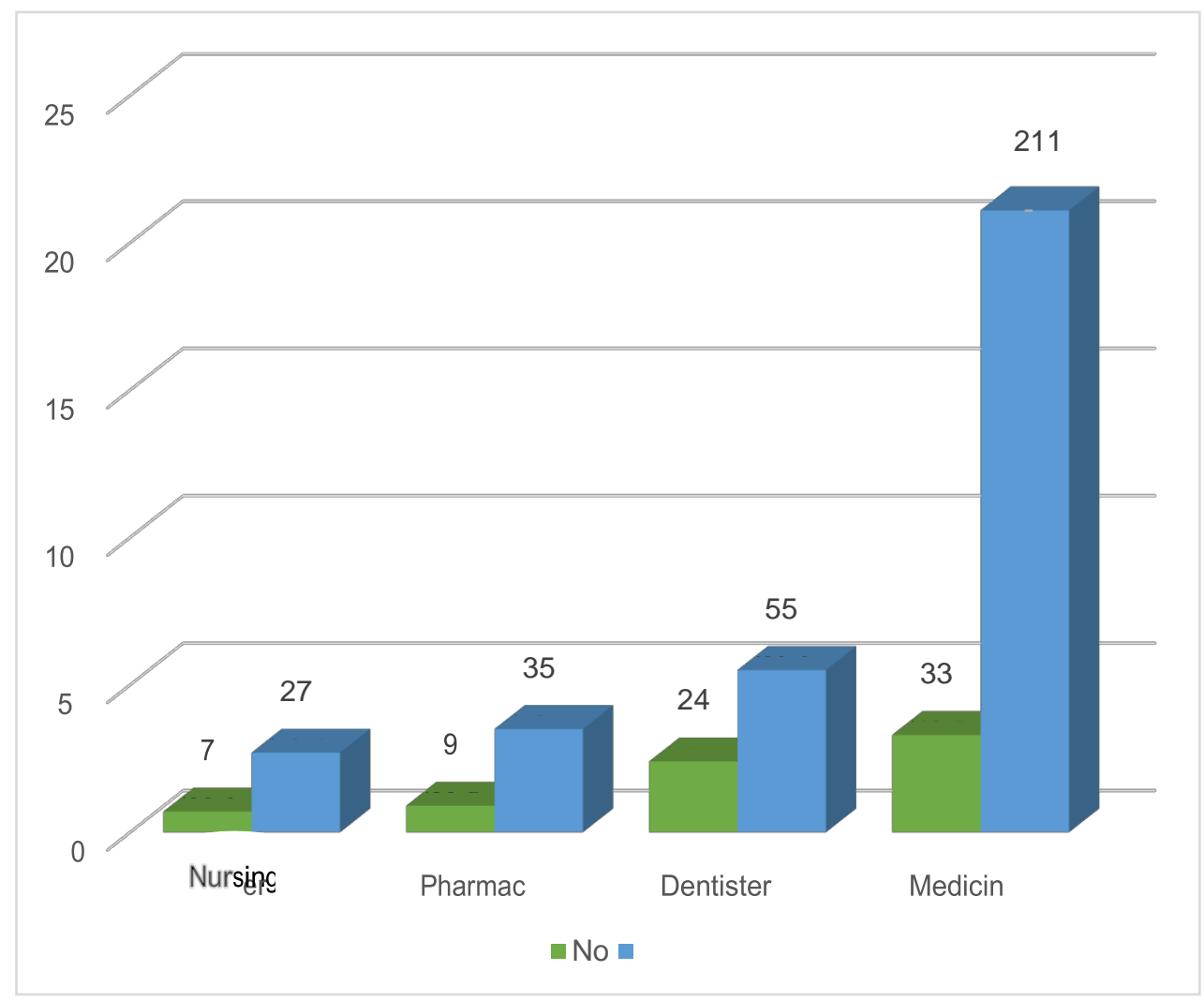

Figure 2: The difference between students in different collages regarding thinking about of healthcare- related infections can be decreased by hands washing 
Table (5) shows the associations between deferent colleges regarding their practice of standard precautions, by asking do they wash their hands before and after each patient care $53.1 \%$ of participants said always and among colleges the highest percentage was in college of nursing $58.8 \%$ of them wash their hands every time they care a patient with statistically not significant p-value (0.433), and about $47.6 \%$ of participants on the same patient between tasks and procedures sometimes wash their hand and the highest percentage was in college of dentistry $51.9 \%$ and statistically non-significant p value (0.607),by asking do they wash their hands before and after using gloves $47.6 \%$ said sometimes which among them the highest percentage was in college of medicine $50.4 \%$ of them said sometimes and statistically non-significant $\mathrm{p}$ value (0.43), regarding dealing with the same patient they re-use the masks and gloves $41.9 \%$ said sometimes ,among them the highest percentage was in college of nursing $50.0 \%$ with statistically not significant p value (0.376), when we asked do they recap the sharp needle before final disposal $44.6 \%$ said always which among them the highest percentage was in college of nursing $61.8 \%$ of them said always and statistically significant p value (0.046),by asking do they use antibiotic after exposure to a patient with flu $39.7 \%$ of participants said sometimes and among them the highest percentage was in college of nursing $47.1 \%$ said sometimes with statistically significant p value (0.008), by asking do they eat food directly after finishing their practice $46.9 \%$ said sometimes and among them the highest percentage was in college of nursing $50.0 \%$ of them said sometimes and statistically non-significant $\mathrm{p}$ value (0.655).

Table 5: Associations between deferent colleges regarding their practice of standard precautions

\begin{tabular}{|c|c|c|c|c|c|c|c|}
\hline \multirow{3}{*}{\multicolumn{2}{|c|}{ Practice }} & \multicolumn{4}{|c|}{ College } & \multirow[t]{2}{*}{ Total } & \multirow{3}{*}{$\begin{array}{c}\text { P. } \\
\text { Value }\end{array}$} \\
\hline & & \multirow{2}{*}{$\begin{array}{c}\text { Medicine } \\
\text { Number } \\
(\%)\end{array}$} & \multirow{2}{*}{$\begin{array}{c}\text { Nursery } \\
\text { Number } \\
\text { (\%) }\end{array}$} & \multirow{2}{*}{$\begin{array}{c}\text { Dentistry } \\
\text { Number } \\
(\%)\end{array}$} & \multirow{2}{*}{$\begin{array}{c}\text { Pharmacy } \\
\text { Number } \\
(\%)\end{array}$} & & \\
\hline & & & & & & $\begin{array}{c}\text { Number } \\
\text { (\%) }\end{array}$ & \\
\hline \multirow{4}{*}{$\begin{array}{l}\text { 1. Do you wash } \\
\text { your hands } \\
\text { before and after } \\
\text { each patient } \\
\text { care? }\end{array}$} & Always & $137(56.1)$ & 20(58.8) & $36(45.6)$ & $20(45.5)$ & 213(53.1) & \multirow{4}{*}{0.433} \\
\hline & Never & $7(2.9)$ & $2(5.9)$ & $4(5.1)$ & $3(6.8)$ & $16(4.0)$ & \\
\hline & Sometimes & $100(41.0)$ & 12(35.3) & $39(49.4)$ & $21(47.7)$ & $172(42.9)$ & \\
\hline & Total & $244(100)$ & $34(100)$ & $79(100)$ & $44(100)$ & $401(100)$ & \\
\hline \multirow{4}{*}{$\begin{array}{l}\text { 2. Do you wash } \\
\text { your hand } \\
\text { between tasks } \\
\text { and procedures } \\
\text { on the same } \\
\text { patient? }\end{array}$} & Always & 94(38.5) & 14(41.2) & 26(32.9) & 13(29.5) & $147(36.7)$ & \multirow{4}{*}{0.607} \\
\hline & Never & $35(14.3)$ & $5(14.7)$ & $12(15.2)$ & $11(25.0)$ & $63(15.7)$ & \\
\hline & Sometimes & $115(47.1)$ & $15(44.1)$ & 41(51.9) & $20(45.5)$ & 191(47.6) & \\
\hline & Total & $244(100)$ & $34(100)$ & 79(100) & $44(100)$ & $401(100)$ & \\
\hline
\end{tabular}




\begin{tabular}{|c|c|c|c|c|c|c|c|}
\hline \multirow{4}{*}{$\begin{array}{l}\text { 3. Do you wash } \\
\text { your hands } \\
\text { before and after } \\
\text { using gloves? }\end{array}$} & Always & 92(37.7) & 18(52.9) & 29(36.7) & $16(36.4)$ & 155(38.7) & \multirow{4}{*}{0.43} \\
\hline & Never & 29(11.9) & 4(11.8) & $12(15.2)$ & $9(20.5)$ & 54(13.5) & \\
\hline & Sometimes & $123(50.4)$ & 12(35.3) & $38(48.1)$ & $19(43.2)$ & 192(47.9) & \\
\hline & Total & $244(100)$ & $34(100)$ & 79(100) & $44(100)$ & $401(100)$ & \\
\hline \multirow{4}{*}{$\begin{array}{l}\text { 4. Do you re- } \\
\text { use the masks } \\
\text { and } \\
\text { gloves while } \\
\text { dealing with the } \\
\text { same } \\
\text { patient? }\end{array}$} & Always & 71(29.1) & $10(29.4)$ & 17(21.5) & 13(29.5) & 111(27.7) & \multirow{4}{*}{0.376} \\
\hline & Never & 74(30.3) & $7(20.6)$ & 31(39.2) & $10(22.7)$ & $122(30.4)$ & \\
\hline & Sometimes & $99(40.6)$ & $17(50.0)$ & $31(39.2)$ & $21(47.7)$ & 168(41.9) & \\
\hline & Total & $244(100)$ & $34(100)$ & 79(100) & $44(100)$ & $401(100)$ & \\
\hline \multirow{4}{*}{$\begin{array}{l}\text { 5. Do you recap } \\
\text { the sharp needle } \\
\text { before final } \\
\text { disposal? }\end{array}$} & Always & $99(40.6)$ & 21(61.8) & $44(55.7)$ & 15(34.1) & $179(44.6)$ & \multirow{4}{*}{0.046} \\
\hline & Never & 51(20.9) & $5(14.7)$ & $9(11.4)$ & $11(25.0)$ & $76(19.0)$ & \\
\hline & Sometimes & $94(38.5)$ & $8(23.5)$ & 26(32.9) & 18(40.9) & $146(36.4)$ & \\
\hline & Total & $244(100)$ & $34(100)$ & $79(100)$ & $44(100)$ & $401(100)$ & \\
\hline
\end{tabular}




\begin{tabular}{|c|c|c|c|c|c|c|c|}
\hline \multirow{4}{*}{$\begin{array}{l}6 . \text { Do you use } \\
\text { antibiotic } \\
\text { after } \\
\text { exposure to a } \\
\text { patient with } \\
\text { flu? }\end{array}$} & Always & $66(27.0)$ & $14(41.2)$ & $35(44.3)$ & 15(34.1) & $130(32.4)$ & \multirow{4}{*}{0.008} \\
\hline & Never & $84(34.4)$ & $4(11.8)$ & 14(17.7) & $10(22.7)$ & 112(27.9) & \\
\hline & Sometimes & $94(38.5)$ & $16(47.1)$ & $30(38.0)$ & 19(43.2) & 159(39.7) & \\
\hline & Total & $244(100)$ & $34(100)$ & $79(100)$ & $44(100)$ & 401(100) & \\
\hline \multirow{4}{*}{$\begin{array}{l}\text { 7. Do you eat } \\
\text { food directly } \\
\text { after finishing } \\
\text { your practice? }\end{array}$} & Always & $69(28.3)$ & 12(35.3) & $24(30.4)$ & $16(36.4)$ & 121(30.2) & \multirow{4}{*}{0.655} \\
\hline & Never & $59(24.2)$ & $5(14.7)$ & $16(20.3)$ & $12(27.3)$ & $92(22.9)$ & \\
\hline & Sometimes & $116(47.5)$ & $17(50.0)$ & $39(49.4)$ & $16(36.4)$ & 188(46.9) & \\
\hline & Total & $244(100)$ & $34(100)$ & $79(100)$ & $44(100)$ & $401(100)$ & \\
\hline
\end{tabular}

Fig(3) by asking do they use gloves while doing physical examination $17.2 \%$ said never and $38.9 \%$ of them said always which among them the highest percentage was in college of dentistry $54.4 \%$ of them said always and in college of nursing $47.1 \%$ said always and statistically significant p value (0.016).

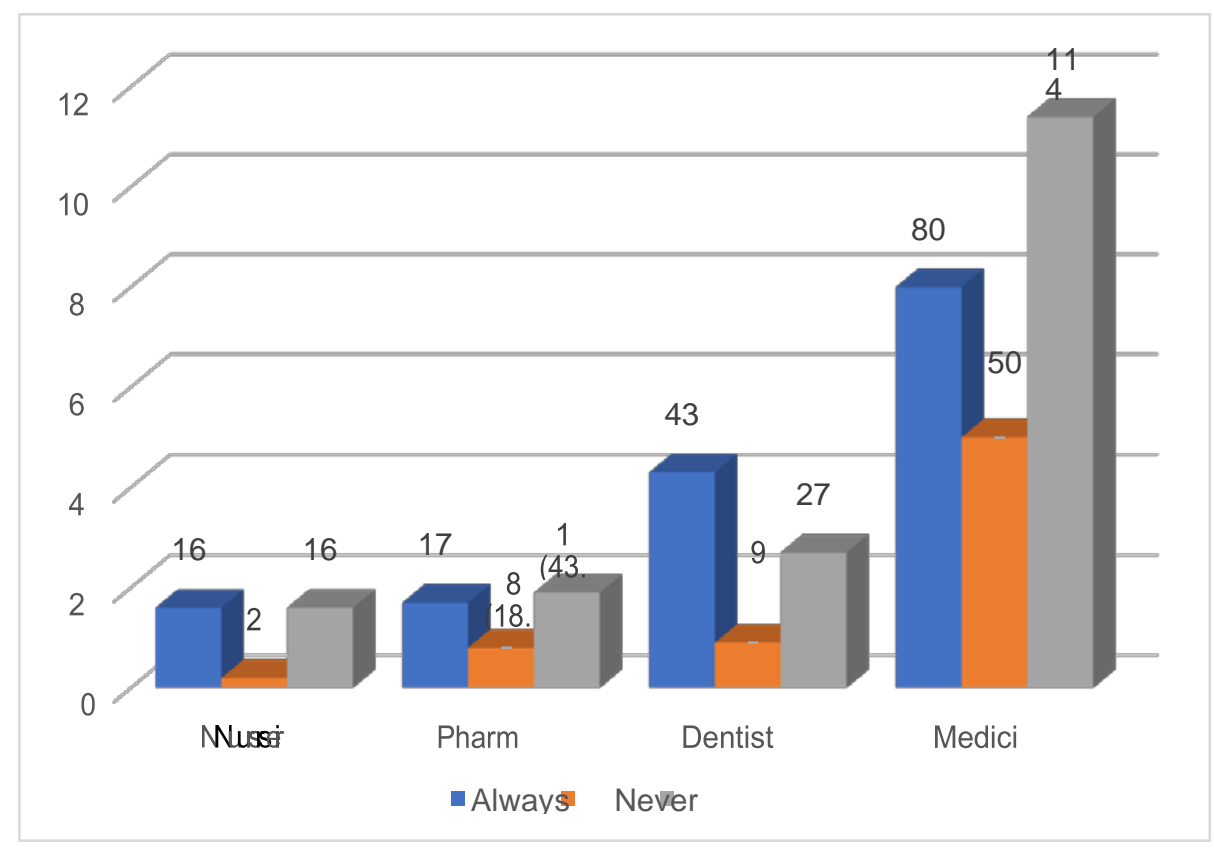

Figure 3: The difference between students in different collages regarding using gloves while doing physical examination 
Fig. (4) by asking have they ever taken any vaccine for hepatitis (B) $43.6 \%$ said no and $56.4 \%$ of participants said yes which among them the highest percentage was in college of medicine $58.8 \%$ of them said yes and statistically non-significant $p$ value $(0.343)$.

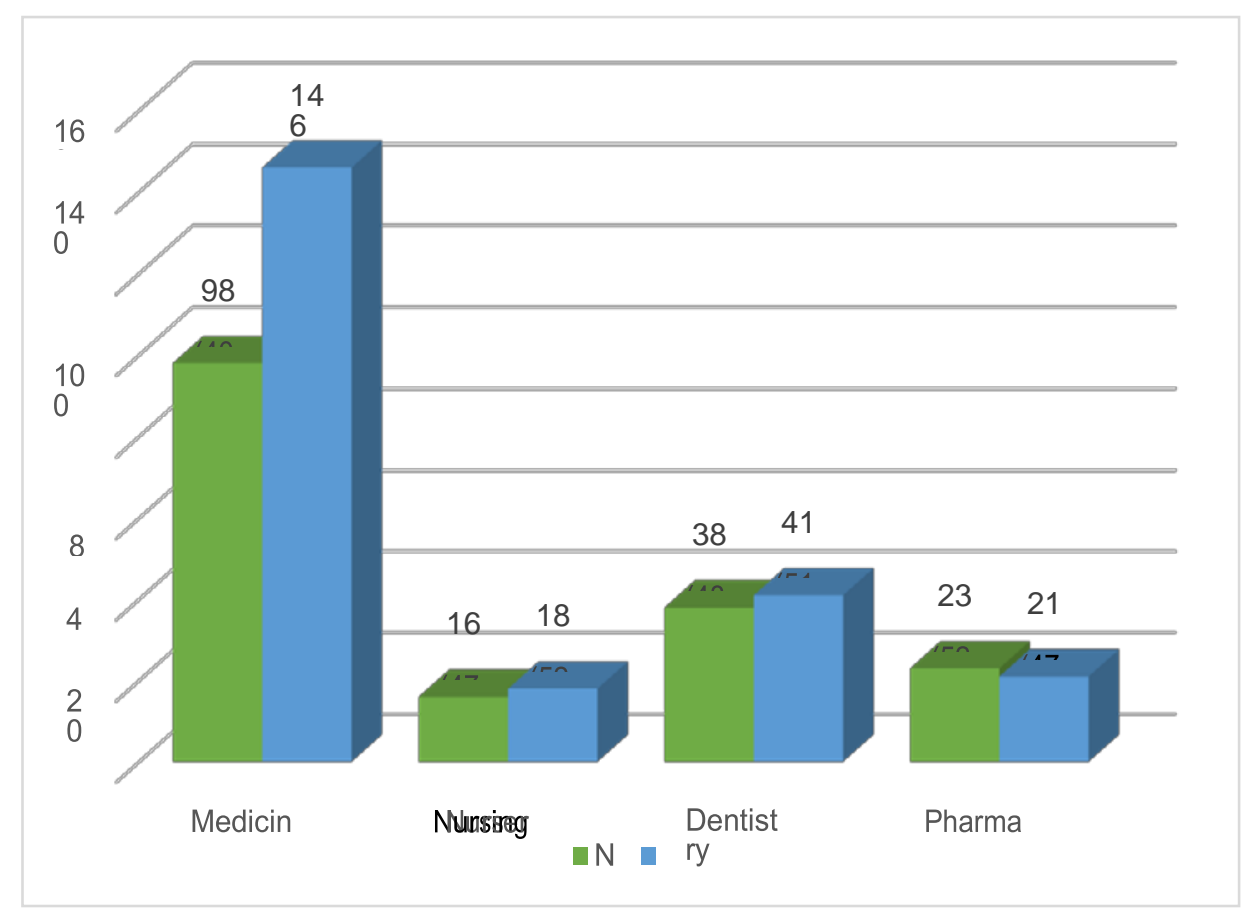

Figure 4: The difference between students in different collages regarding taking vaccine for hepatitis B

\section{DISCUSSION}

In this study the number of the participant was 401 and the mean age was $(21.33 \pm 1.898)$, in a comparison with a study done among medical students in Shiraz, Iran ${ }^{11}$. the main age of the participants in this study was lesser than their which's (24.92 \pm 2.88$)$ it may be due to the difference for acceptance in university of Sulaimani and Shirzad, Regarding the Gender $50.4 \%$ of of the participants was female this goes with a study done in shiraz-Iran in which $38.1 \%$ of their participants was female ${ }^{12}$, this difference could be due to that females in this study were more cooperative. Regarding Knowledge of the participants $80.4 \%$ of students thought after any contact with blood hands should be washed and this goes with a research in medical student in Iran $^{11}$ in which $100 \%$ thought so this is may be due to in here first and second stages have more time to fill the forms and they don't know about precaution yet , In this study $79.3 \%$ of the students said in touching mucous membrane gloves should be worn and $90.5 \%$ of medical students in Iran ${ }^{11}$ research said so, in this study $68.1 \%$ of students 
thought to protect eyes, protective glasses should be worn but $91.2 \%$ of students in $\operatorname{Iran}^{11}$ research said protective glasses should be worn may be because students in this study look at their senior doctors and they don't wear it so they think that it is not important. In this study $67,1 \%$ students said grown is necessary when entering room patient and this goes with Iranian research ${ }^{11}$ which $71.6 \%$ students said same , $48.1 \%$ of students thought needles must be bent before disposal and $62.4 \%$ in Iran ${ }^{11}$ research said same this difference due to students don't read the form carefully but they fill it without reading the questions. In this study $75.6 \%$ said all health providers are at risk for infection and this goes with a study in Saudi ${ }^{13}$ in which $75.3 \%$ of the students thought so, regarding students believes $75.6 \%$ of students believed all body fluids except sweat are sources of infection but in a Saudi research ${ }^{13}$ in which $31.9 \%$ said the same, about $24.7 \%$ of the students in this study believed health care provider should receive influenza while in Saudi research $42.2 \%$ believed the same, regarding tuberculin test $27.3 \%$ of participants believed health providers should be tested by tuberculin test and this goes with Saudi research in which $31.5 \%$ believed the same and the same results which were found in tow studies in India ${ }^{14,15}$,in this study $36.7 \%$ of students said hands should be washed after procedures on the same patient and in Saudi research $47.4 \%$ said so ${ }^{13}$. For the immunization $20.2 \%$ of participants thought routine immunization for HIV and rubella is necessary to all health provider and $24.7 \%$ of participants of Saudi ${ }^{16}$ research thought so , and about $56.4 \%$ in this study thought whatever the diagnosis is all patients can be sources of infection this goes with a Saudi research ${ }^{16}$ in which $41.8 \%$ thought the same. In this research $53.1 \%$ of students before and after each patient examination they always wash their hands and this goes with a research in Saudi Arabia ${ }^{16}$ in which $68.6 \%$ always wash their hands this is may be due to that these students were more educated, in this study $37.7 \%$ always wash their hand before and after wearing gloves and $44.6 \%$ always recap needles before final disposal this goes in contrast with a study in Saudi ${ }^{16}$ in which $17.4 \%$ of students always recap needles and goes with a study in $\mathrm{UK}^{17}$. About $32.4 \%$ always receive antibiotic after exposure to a patient with flu and in a study of Saudi Arabia $20.4 \%$ only receive antibiotic ${ }^{16}$. Regarding change their gloves between different tasks on the same patient about $47.6 \%$ of the participants answered yes this goes with a Saudi research ${ }^{16}$ in which $47.4 \%$ of their students change their gloves, About the Hepatitis B Vaccine $56.4 \%$ of the participants were vaccinated with hepatitis B vaccine and this goes with a study done in Saudi Arabia that $52.2 \%$ of participants were vaccinated ${ }^{18}$, this compares with the zero to $22.4 \%$ observed in other studies in Nigeria among health workers, but contrasts sharply with the over 95\% seen in Australian medical and dental students, $86 \%$ in German medical students, $42.2 \%$ medical students in Pakistan $^{19-25}$. this may be due to lack of information about HBV vaccination advantage among the students in this study.

\section{CONCLUSIONS}

Most of students knew that after any contact with secretions, contaminated item, blood and bloody fluid hands should be washed, and when touching mucous membranes or non-intact skin gloves should be worn with highest percentage from college of nursing. Nearly half of the students aware about bending of needle before disposal. Majority thought that all patients can transmit infections whatever their diagnosis are, believed that all healthcare workers are at risk of infections during their work and healthcare-related infection can be decreased by hand washing. Most of the students believed that no need for hand washing if they use gloves, Only sweat shouldn't be regarded as sources of infection, before and after each patient care always wash their hands and have taken a vaccine for Hepatitis B. During tasks and procedures on the same patient less than half of students wash their hands, and before final disposal of the sharp needles they always recap them, always use gloves while doing physical examination. Less than quarter of students never eat food directly after finishing their practice. 


\section{REFERENCES}

[1] AR Isara, AN Ofili, "Knowledge and practice of standard precautions among health care workers in the Federal Medical Centre Asaba Delta State Nigeria”, Niger Postgrad Med J , Sep17(3), pp 204-9, 2010.

[2] Askarian, M., Honarvar, B., Tabatabaeeb, H. R , "Knowledge, attitude and practice towards standard isolation precautions in Iranian medical students”, J Hosp Infect, December 58(4), pp 292-296, 2004.

[3] Cincinnati, OH, "preventing needle stick injuries in health care settings", Department of Health and Human Services, CDC, DHHS publication (NIOSH), 108, 2000.

[4] G Phillips, J Ker, "Champion students Experience with a standardized infection control training package in medical students” J Hosp Infect, April, 62 (4) , 518-519, 2006.

[5] V Wiwanitkit, "Knowledge survey concerning universal precaution among the Thai preclinic year medical students: a medical school-based study”, Am J Infect Control, June, 30(4) , 255-256, 2002.

[6] Malekzadeh, R., Azizi, F., M.Janghorbani , "Viral hepatitis epidemiology and control of common disorders in Iran”, Tehran, Eshtyagh , pp. 717-718, 2001.

[7] Elliott, S. K. F., Keeton, A., Holt A. "'Medical students’ knowledge of sharps injuries” , J Hosp Infect, , 60(4), 374-377, 2005.

[8] Askarian, M., Honarvr, B., Tabatabaee, H. R., Assadian O. , "Knowledge, practice and attitude towards standard isolation precaution in Iranian medical students”, J Hosp Infect, December,58(4), 292-296, 2004.

[9] A.Feather, S. P.Stone., A.Wessier, K. A. Boursicot, C. Pratt ,"Now please wash your hands': the hand washing from: http behavior of final MBBS candidates”, J Hosp Infect, May, 45(1), 62-64, 2000.

[10] Hunt, D. C. E., A.Mohammudaly, S. P.Stone , J.Dacre , "Hand hygiene behaviour, attitudes and beliefs in first year clinical medical students”. J Hosp Infect, April, 59( 4) , 371-373, 2005.

[11] B Ameneh, A Ahmad, "Knowledge Attitude and Practice towards Standard Isolation Precautions among Iranian Medical Students”, Global Journal of Health Science, March,4(2),142-147, 2012.

[12] M. Askarian, K.Aramesh, Ch.J. Palenik. "Knowledge attitude and practice toward contact isolation precautions among medical students in Shiraz Iran”, American journal of infection control, November,34(9) ,593- 596, 2006.

[13] MM Alotaibi, SM Almasari, AN Alkadam, YA Alanazi, KA Gahtani. "Knowledge and compliance with standard isolation precautions among healthcare students in Al-Kharj Governorate”, Saudi Arabia. J Health, Spec 5, pp162-70, 2017.

[14] A Kotwal, DK Tareja, "Healthcare workers and universal precautions perception and determinants of noncompliance”, Indian J Community Med, 35(4) ,pp551-553, 2010.

[15] M Kermode, D Jolley, M Langkhan, D Thomas M.S, N Crofts, “Occupational exposure to blood and risk of blood borne viruses infection among healthcare workers in rural North Indian healthcare settings”, An Infect Control. 33(1),pp34-41, 2005.

[16] T Amin. T. , Kh Al Noaim, I. , M. A Bu Saad., T. A.Al Malhm , A. A. Al Mulhim, M. A. A. A. Al Awas, "Standard Precautions and Infection Control Medical Students Knowledge and Behavior at a Saudi University the Need for Change”, Global Journal of Health Science, 5(4), pp114-125, 2013.

[17] Elliott, SKF, A Keeton, A. A Holt , “Medical students' knowledge of sharps injuries” , J Hosp Infect 60, pp374-377, 2005.

[18] O. Al Wutayd , A AlRehaili , Kh. AlSafrani , A. Abalkhail, S. M. AlEidi, "Current Knowledge, Attitudes and Practice of Medical Students Regarding the Risk of Hepatitis B Virus Infection and Control Measures at Qassim University”, Journal of Medical Sciences, Feb 7(3), pp 435-439, 2019.

[19] I O Olubuyide , S O Ola , B Aliyu ,” Prevalence and epidemiological characteristics of hepatitis B and C infections among doctors and dentists in Nigeria”, East Afr Med J , 74, pp357-361, 1997.

[20] CA Ademowo, A. Ajuwon, "The immunization status and level of knowledge about hepatitis B virus infection among Nigerian surgeons”, West Afr J Med, 16 pp93-96, 1997.

[21] O O Odusanya , “Awareness and compliance with universal precaution among health workers at an emergency service in Lagos, Nigeria”, Nig Med J, 44, pp13-16, 2003.

[22] RC Ibekwe, N Ibeziako, “Hepatitis B vaccination status among health workers in Enugu” , Nigeria. Nig j Clin Pract, 9, pp7- 10, 2006.

[23] DR C R RC Smith, PA Leggat, “Needle stick and sharp injuries among nursing students” , J Adv Nursing , 51, pp449-455, 2005.

[24] S Deisehammer, K Radon, D Nowak, J Reichert, “Needle stick injuries medical training” , J Hosp Infect 63, pp 263-267, 2007.

[25] K Nasir, KA Khan, WM Kadiri, "Hepatits B vaccination among health care workers and students of a medical college”, J Pak Med Assoc, 50, pp239-243, 2000. 